DOE/ER/40785-3

\title{
RESEARCH IN THEORETICAL NUCLEAR PHYSICS
}

\author{
Final Report
}

April 1, 1993 - March 31, 1996

Takeshi Udagawa

Theoretical Nuclear Physics Group

Department of Physics

The University of Texas at Austin

P. O. Box 7726, Austin, Texas, 78713-7726

August 1997

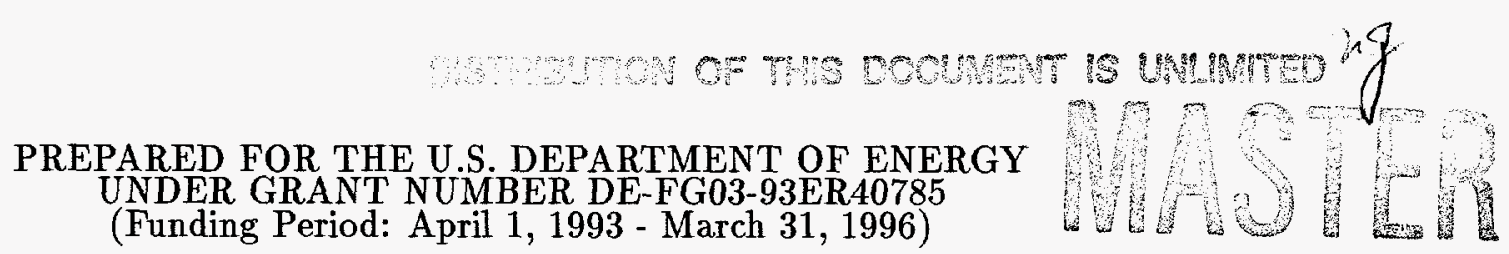

\section{DISCLAIMER}

This report was prepared as an account of work sponsored by an agency of the United States Government. Neither the United States Government nor any agency thereof, nor any of their emplcyees, makes any warranty, express or implied, or assumes any legal liability or responsibility for the accuracy, completeness, or usefulness of any information, apparatus, product, or process disclosed, or represents that its use would not infringe privately owned rights. Reference herein to any specific commercial product, process, or service by trade name, trademark, manufacturer, or otherwise does not necessarily constitute or imply its endorsement, recommendation, or favoring by the United States Government or any agency thereof. The views and opinions of authors expressed herein do not necessarily state or reflect those of the United States Government or any agency thereof. 


\section{DESCLAMER}

Portions of this docoment may be illegible in electronic imsge products. Imsges are produced from the best available original doceromert 


\section{Introduction}

This report describes the accomplishments in basic research in nuclear physics carried out by the theoretical nuclear physics group in the Department of Physics at the University of Texas at Austin, during the period of April 1, 1993 to March 31, 1996. The work done covers three separate areas, low energy nuclear reactions, intermediate energy physics, and nuclear structure studies. Although the various subjects are spread among different areas, they are all based on two techniques that we have developed in previous years. These techniques are: a) a powerful method for continuum-random-phase-approximation (CRPA) calculations of the nuclear response; ${ }^{1}$ and, b) the direct reaction approach ${ }^{2,3}$ to complete and incomplete fusion reactions, which enables us to describe on a single footing all the different types of nuclear reactions, i.e., complete fusion, incomplete fusion and direct reactions, in a systematic way based on a single theoretical framework. In what follows, we first summarize our achievements in these three areas, and then present final remarks.

\section{Low Energy Nuclear Reactions}

The research in low energy nuclear reactions has covered the following two subject: development of a fast computer code for coupled-channel (CC) calculations of heavy ion scattering, and its applications to a simultaneous analysis of data of heavy ion scattering and fusion at near- and sub-Coulomb barrier energies, and spectroscopic analyses of transfer reactions leading to both bound and unbopund final states of the residual nucleus.

The development of a computer code for CC calculations of heavy ion scattering was accomplished by using a technique developed for the CRPA nuclear response calculations. ${ }^{1}$ The method has turned out to be very efficient, speeding up the computation time by a large factor. This factor becomes even larger as the dimension of the coupled equation increases and thus can easily become an order of magnitude, if the dimension of the coupled-channel equations reaches about 100 .

Using the code, an extensive analysis has been carried out ${ }^{4}$ of experimental data of elastic 
and inelastic scattering ${ }^{5}$ and fusion cross sections ${ }^{6}$ for the ${ }^{16} \mathrm{O}+{ }^{152,154} \mathrm{Sm}$ and ${ }^{16} \mathrm{O}+{ }^{186} \mathrm{~W}$ systems at sub- and near-Coulomb barrier energies. The fusion process is treated by means of a fusion potential, which is a part of the imaginary piece of the optical potential. The idea of describing fusion in terms of a fusion potential was developed in the course of our work on a unified approach to nuclear reactions. ${ }^{2}$ We took into account the lowest rotational states of the target, up to $I^{\pi}=8^{+}$, explicitly in the calculation. It is shown that experimental data of fusion cross sections taken recently with high precision, ${ }^{6}$ as well as partial fusion cross sections ${ }^{7}$ and also average spin values of compound nuclei formed after fusion, ${ }^{8}$ are well reproduced by the calculations using optical potential parameters that can reproduce elastic and inelastic scattering data. The so-called fusion-barrier distribution, defined as the second derivative of the fusion cross section $d^{2}\left(E \sigma_{F}\right) / d E^{2}$, is also calculated and compared with experimental data. ${ }^{6}$ The calculations were able to explain successfully the characteristic difference in the distributions between the ${ }^{16} \mathrm{O}+{ }^{154} \mathrm{Sm}$ and ${ }^{16} \mathrm{O}+{ }^{186} \mathrm{~W}$ systems in terms of the difference in the sign of $\beta_{4}$ in the above two systems.

The spectroscopic analyses of the transfer reactions leading to bound and unbound final nuclear states were carried out by using the breakup-fusion (BF) method $^{3}$ for the ${ }^{40} \mathrm{Ca}\left({ }^{3} \mathrm{He}, d\right)$ and ${ }^{40} \mathrm{Ca}\left({ }^{3} \mathrm{He}, d p\right)$ reactions with incident energy of $E=240 \mathrm{MeV}$ as well as the ${ }^{40} \mathrm{Ca}\left({ }^{6} \mathrm{Li}, \mathrm{d}\right){ }^{44} \mathrm{Ti}$ reaction with $\mathrm{E}=60 \mathrm{MeV}$. The $\mathrm{BF}$ method was originally developed for calculating cross sections of incomplete-fusion reactions to the highly excited continuum. ${ }^{3}$ It has been shown ${ }^{9}$ that the method can also be used for spectroscopic purposes, i.e., for analyzing data of nuclear transfer reactions. The merit of the method, as compared with the usual DWBA method, is that it provides a unified description of the reactions to both bound and unbound final states. The unification is achieved by utilizing the complex optical potential for the transfered particle even in the bound state, instead of utilizing the real potential used in the usual DWBA method. The BF method also enables us to calculate the so-called background cross section on the same footing as the transfer cross sections are evaluated; in the conventional analysis with the DWBA method, the background component 
is subtracted from the observed cross section in a rather arbitrary manner, which inevitably introduces an ambiguity in the analysis. With the BF method, one can thus carry out the analyses without such an ambiguity.

The main aim of the analyses was to extract information on single particle and cluster structure of the residual nuclei in the bound and unbound regions. For the ${ }^{40} \mathrm{Ca}\left({ }^{3} \mathrm{He}, \mathrm{d}\right)^{41} \mathrm{Sc}$ reaction, we were able to deduce ${ }^{10}$ fairly conclusive information on the distribution of the $f_{5 / 2}$

and $g_{9 / 2}$ single particle strength in the unbound region and also the $f_{7 / 2}$ and $p_{3 / 2}$ strength in both bound and unbound regions. In the case of the ${ }^{40} \mathrm{Ca}\left({ }^{6} \mathrm{Li}, \mathrm{d}\right){ }^{44} \mathrm{Ti}$ reaction, we were able to identify ${ }^{11}$ for the first time all $0^{+}-12^{+}$members of the $\alpha$-cluster states of the principal quantum number $\mathrm{N}=12$ band. The spectroscopic factors of these states were extracted to be about $30 \%$ of the sum rule limit, thus indicating that the cluster strength is largely spread into other states. We could then identify most of the states into which the strength was spread. Another important result obtained is that we could identify a strong candidate for the $0^{+}$band head of the $\mathrm{N}=14$ cluster band at an excitation energy of $9.32 \mathrm{MeV}$. The successful results have given us a great deal of confidence in the validity of the BF method as a spectroscopic tool.

\section{Intermediate Energy Physics}

The work done in the area of intermediate energy physics is concerned with a theoretical method for dealing with nuclear medium effects on the delta $(\Delta)$ excited in nuclei by means of hadronic charge-exchange reactions as well as $\gamma$ - and $\pi$-absorption at intermediate energies. Using our technique for solving the CRPA equation, ${ }^{1}$ we have developed a method for calculating both inclusive and exclusive cross sections of these reactions, in which all important nuclear medium effects, i.e., those of one body mean fields for the target nucleons as well as the excited $\Delta$, two-body $\Delta$-hole interactions, and $\Delta$ spreading, are taken into account. It has been shown that the calculations done by using the method reproduce the observed inclusive cross sections for charge-exchange ${ }^{12} \mathrm{C}(p, n)$ and $\left({ }^{3} \mathrm{He}, t\right)$ 
reactions very well, ${ }^{12,13}$ except at lower energies, i.e., in the so-called gap and slightly above gap regions. The calculations have also been successful in reproducing data of $\gamma$ - and $\pi$ induced reactions ${ }^{14}$ as well as the exclusive coherent $\pi$-production cross section data taken at SATURN ${ }^{15}$ for the ${ }^{12} \mathrm{C}\left({ }^{3} \mathrm{He}, \mathrm{t} \pi\right)^{12} \mathrm{C}$ reaction. ${ }^{16}$ We have further analyzed data of the spinlongitudinal (LO) and spin-transverse (TR) cross sections, $\sigma_{L O}$ and $\sigma_{T R}$, deduced from the spin-transfer measurements in the ${ }^{12} \mathrm{C}(\vec{p}, \vec{n})$ reaction. ${ }^{17}$ It has been observed that their measured $\sigma_{L O}$ agrees well with our prediction over the entire energy range, where $\sigma_{L O}$ has an appreciable value, while the measured $\sigma_{T R}$ agrees with our theoretical prediction at higher excitation energy region, roughly higher than the resonance peak, but the fit becomes worse at lower excitation energies. The discrepancy at the lower excitation energies is reminiscent of the discrepancy seen in the inclusive cross section. This means that the trouble we have seen in the inclusive cross section is due to our theoretical calculations underestimating the spin TR cross section at the low excitation energy region.

The above discrepancy occurs only in the nuclear target case, but not in the proton target case. This seems to suggest that origins of the trouble may be ascribed to the nuclear many body current effects, which have not been taken into account in calculations made so far. The most intriguing, but unexplored nuclear many body current, specifically associated with the spin TR excitations, is the two-body current due to the $\rho$-meson exchange. Chang Lee, one of our graduate students, worked on this problem, taking the $d(p, n) p p$ reaction as an example, demonstrating that it is indeed possible to remove the discrepancy by taking into account the two-body current effect.

Another interesting finding made in the spin transfer coefficient measurements ${ }^{17}$ is the existence of a non-vanishing $\Delta S=0$ component, $\sigma_{0}$, in the inclusive cross section. Within the theoretical framework we use now, we predict $\sigma_{0}=0$. One conceivable mechanism that produces a non-vanishing $\sigma_{0}$ is the projectile $\Delta$-excitation (PDP) mechanism. Yung Jo, one of the graduate students of our group, and Chang Lee estimated the contribution from this process to $\sigma_{0}$, finding that the observed $\sigma_{0}$ can be very well explained in terms of a 
projectile excitation mechanism. ${ }^{19}$

\section{Nuclear Structure}

An important advantage of our continuum random-phase-approximation method, developed in Ref. 1, is that it enables us to calculate contributions from individual particle-hole (ph) components to the total strength function. This allows us to calculate the particle emission decay probabilities, $(\gamma, p)$ and $(\gamma, n)$ cross sections, and also a variety of coincidence cross sections. One of the studies made along this line was a calculation of emission widths of the isobaric analogue (IA) and Gamow-Teller (GT) resonance states in ${ }^{208} \mathrm{~Pb}$. We were very successful ${ }^{20}$ in reproducing the observed partial escape widths for both IA and GT resonance that were deduced from very recent coincidence measurements ${ }^{21}$ of the $\left({ }^{3} \mathrm{He}, t p\right)$ reaction with an incident energy of $E=450 \mathrm{MeV}$. The CRPA method can treat the continuum effects in an exact manner, which was found to be necessary to make reliable predictions of the emission widths. We have also made a study ${ }^{22}$ of the $(\gamma, p)$ and $(\gamma, n)$ reactions cross sections in collaboration with Dr. Gomez-Chamacho, an ex-student of our theoretical group, now a research fellow at Instituto National de Investigaciones Nuclerares, Mexico.

\section{Final Remarks}

The work done by the theoretical nuclear physics group in the Department of Physics at the University of Texas at Austin during the period of April 1, 1993 to March 31, 1996, covers three separate areas, low energy nuclear reactions, intermediate energy physics, and nuclear structure studies. Although the various subjects are spread among different areas, they are all based on two techniques that we have developed in previous years. The research was concluded very successfully; we published during the period 23 papers that are summerized in the list of publication attached. 
1. T. Udagawa and B. T. Kim, Phys. Rev. C 40, 2271 (1989).

2. T. Udagawa, T. Tamura, and B. T. Kim, Phys. Rev. C 39, 1840 (1989).

3. T. Udagawa, X.-H. Li, and T. Tamura, Phys. Rev. C 37, 429 (1988).

4. T. Izumoto, T. Udagawa, and B. T. Kim, Phys. Rev. C. 51, 761 (1995).

5. R. G. Stokstad and E. E. Gross, Phys. Rev. C 23, 281 (1981).

C. E. Thorn et al., Phys. Rev. Lett. 38, 384 (1977).

P. Talon et al., Nucl. Phys. A359, 493 (1981).

6. J. X. Wei et al., Phys. Rev. Lett. 67, 3368 (1991).

R. C. Lemmon, et al., Phys. Lett B 316, 32 (1993).

7. A. H. Wuosmaa et al., Phys. Lett. B 263, 23 (1991).

8. S. Gil et al., Phys. Rev. C 43, 701 (1991).

9. T. Udagawa, Y. J. Lee, and T. Tamura, Phys. Rev. C 39, 1 (1989).

10. C.-Y. Kim et al., Phys. Rev. C 50, 2035 (1994).

11. C.-Y. Kim et al., Nucl. Phys. A564, 425 (1993).

12. T. Udagawa, S.-W. Hong, and F. Osterfeld, Phys. Lett. B 245, 1 (1990).

13. T. Udagawa et al., Phys. Rev. C. 48, 3162 (1994).

14. B. Koerfgen et al., Phys. Rev. C 50, 1637 (1994).

15. J. Hennino et al., Phys. Lett. B 303, 236 (1993).

16. P. Oltmanns, F. Osterfeld, and T. Udagawa, Phys. Lett. B 299, 194 (1993).

17. D. Prout et al., Phys. Rev. Lett. 76, 4488 (1996).

18. C.-Y. Lee, Phys. Rev. C 55, 349 (1997).

19. Y. Jo and C.-Y. Lee, Phys. Rev. C 54, 952 (1996).

20. T. Udagawa, D. Knobles, and S. Stotts, Nucl. Phys. A577, 67c (1994) and Phys. Rev. C bf 52,2257 (1995).

21. H. Akimune et al., Phys. Lett B 323, 107 (1994).

22. A. Gomes and T. Udagawa, Nucl. Phys. A565, 607 (1993). 
" $\Delta$ Excitation in Nuclei and the Coherent Pion Decay", F. Osterfeld, B Koerfgen, P. Oltmanns, and T. Udagawa, Physica Script 48, 95 (1993).

"Nuclear Response in the Continuum: From Giant Resonance to $\Delta$ Excitation Region", T. Udagawa, Proceedings of the International Symposium on Spectroscopy and Structure of Molecule and Nuclei, ed. by N. R. Johnson, W. N. Sheldon, and M. E1-Sayed (World Scientific, 1992), p. 211.

"Coherent Pion Production in Intermediate Energy Charge-Exchange Reactions", P. Oltmanns, F. Osterfeld, and T. Udagawa, Phys. Lett. B 299, 194 (1993).

"Comments on $(\mathrm{p}, \mathrm{n})$ Reactions in the $\Delta$-Resonance Region", T. Udagawa, F. Osterfeld, and P. Oltmanns, Proceedings of Workshop on $N-N$ and $N$-Nucleus Scattering, ed. by T. Taddeucci, M. McNaughton, and J. Ullmann (Los Alamos National Laboratory, 1993) p. 175.

"Coherent Pion Production at Small Scattering Angles", F. Osterfeld, B. Koerfgen, P. Oltmanns, and T. Udagawa, Proceedings of 105th Intern. WE-Heraceus-Seminar on Hadronic Processes at Small Angles in Storage Rings, ed. by E. Roessle and O. W. B. Schult (Konferenzen des Forschungszentrums, Juelich, 1993) p.83.

"Study of the ${ }^{40} \mathrm{Ca}\left({ }^{6} \mathrm{Li}, \mathrm{d}\right){ }^{44} \mathrm{Ti}$ Reaction at $60.1 \mathrm{MeV}$ with the Breakup-Fusion Model", $\mathrm{P}$. Guazzoni, M. Jaskola, L. Zetta, Chong-Yeal Kim, T. Udagawa, and G. Bohlen, Nucl. Phys. A564, 425 (1993).

"Direct Reaction Approach to Heavy Ion Scattering at Energies near Coulomb Barrier", T. Udagawa, Proceedings of the RIKEN International Workshop on Heavy-Ion Reactions with Neutron-Rich Beams", ed. by M. Ishihara, N. Takigawa, and S. Yamaji (World Scientific, 1993), p. 69.

"Calculations on the Giant-Dipole Resonance in the Continuum Random-Phase Approximation with a Momentum-Dependent Interaction", A. Gomez and T. Udagawa, Nucl. Phys. A565, 607 (1993).

"Coherent Pion Production in the Delta Resonance Region", P. Oltmanns, K. Koerfgen, F. Osterfeld, and T. Udagawa, Acta Physica Polonica B 24, 1609 (1993).

"Theoretical Analyses of Inclusive and Exclusive Spectra of Charge-Exchange Reactions in the Delta Resonance Region", T. Udagawa, P. Oltmanns, K. Koerfgen, and F. Osterfeld, Proceedings of the RIKEN International Workshop on Delta Excitations in Nucle $"$, ed. by M. Ishihara, M. Ichimura, and H. Toki (World Scientific, 1994), p. 141.

" $\Delta$ Excitations in Nuclei and their Decay Properties", T. Udagawa, P. Oltmanns, F. Osterfeld, and S. W. Hong, Phys. Rev. C 48, 3162 (1994). 
"Longitudinal and Transverse Spin Response of ${ }^{12} \mathrm{C}$ in the $\Delta$ Resonance Region", B. Koerfgen, F. Osterfeld, and T. Udagawa, Phys. Rev. C 50, 1637 (1994).

"Breakup-Fusion Analyses of the $240 \mathrm{MeV}{ }^{40} \mathrm{Ca}\left({ }^{3} \mathrm{He}, d\right)$ and ${ }^{40} \mathrm{Ca}\left({ }^{3} \mathrm{He}, d p\right)$ Reactions", C.-Y. Kim, T. Udagawa, J. Guillot, H. Langevin-Joliot, J. Van de Wiele, J. J. Florent, A. Willis, E. Hourani, E. Gerlic, and G. Duhamel-Chretien, Phys. Rev. C 50, 2035 (1994).

"Continuum Tamm-Dancoff-Approximation Calculations of the Escape Widths of the GamowTeller Resonance in ${ }^{208} \mathrm{Bi}^{\prime}$, T. Udagawa, D. P. Knobles, and S. A. Stotts, Nucl. Phys. A577, 67c (1994).

" $\Delta$ Excitations in Nuclei and Their Decay Properties", F. Osterfeld, B. Koerfgen, P. Oltmanns, and T. Udagawa, Nucl. Phys. A577, 237c (1994).

"Importance of the Lanczos Method in the Study of Giant Resonances", A. Gomez and T. Udagawa, Revista Mexicana de Fisica 40, Suplemento 1, 91 (1994).

"Coupled-Channel Analyses of Scattering and Fusion Cross Sections of ${ }^{16} \mathrm{O}+{ }^{152,154} \mathrm{Sm},{ }^{186} \mathrm{~W}$ Systems at Sub- and Near-Coulomb Barrier Energies", T. Izumoto, T. Udagawa, and B. T. Kim, Phys. Rev. C 51, 761 (1995).

"Coupled-Channel Calculations of Scattering and Fusion Reactions of ${ }^{16} \mathrm{O}+{ }^{152,154} \mathrm{Sm},{ }^{186} \mathrm{~W}$ Systems at Sub- and Near-Coulomb Barrier Energies", T. Izumoto, B. T. Kim, and T. Udagawa, Proceedings of the International Symposium on Heavy Ion Fusion, ed. by M. Stefanini, G. Nebbia, S. Lunardi, G. Montagnoli, and A. Vitturi (World Scientific, 1995), p. 91.

"Meson Scattering in the Medium", F. Osterfeld, B. Koerfgen, P. Oltmanns, and T. Udagawa, Proceedings of the International Conference on Physics With GeV-Particle Beams, ed. by H. Machner and K. Sistemich, (World Scientific, 1995), p. 389.

"Continuum Tamm-Dancoff Approximation Calculations of the Escape Widths of the Isobaric Analog State and Gamov-Teller Resonance in ${ }^{208}$ Bi, Phys. Rev. C 52, 2257 (1995).

"Coherent Pion Production in Charge-Exchange Reactions at Intermedium Energies, Revista Mexicana de Pisica, Suplemento 1, 72 (1995).

"Spin Decomposition of the $\Delta$ Resonance Cross Section Using the ${ }^{12} \mathrm{C}(\vec{p}, \vec{n})$ Reaction at $E_{p}=795 \mathrm{MeV}$ ", D. L. Prout et. at., Phy. Rev. Lett. 76, 4488 (1996). 